\title{
Distinctive Quantal Properties of Neurotransmission at Excitatory and Inhibitory Autapses Revealed Using Variance-Mean Analysis
}

\author{
Kaori Ikeda, ${ }^{1}$ Yuchio Yanagawa, ${ }^{2}$ and John M. Bekkers ${ }^{1}$ \\ ${ }^{1}$ Division of Neuroscience, The John Curtin School of Medical Research, The Australian National University, Canberra, Australian Capital Territory 0200, \\ Australia, and 2Department of Genetic and Behavioral Neuroscience, Gunma University Graduate School of Medicine, Maebashi 371-8511, Japan
}

\begin{abstract}
Normal brain function depends on an interplay between glutamatergic and GABAergic synaptic transmission, yet questions remain about the biophysical differences between these two classes of synapse. By taking advantage of a simple culture system, we present here a detailed comparison of excitatory and inhibitory neurotransmission under identical conditions using the variance-mean $(V-M)$ method of quantal analysis. First, we validate $V-M$ analysis for glutamatergic autapses formed by isolated hippocampal pyramidal neurons in culture, confirming that the analysis accurately predicts the quantal amplitude $(Q)$. We also show that $V-M$ analysis is only weakly sensitive to intersite and intrasite quantal variance and to the known inhomogeneities in release probability $\left(P_{\mathrm{r}}\right)$. Next, by repeating the experiments with GABAergic autapses, we confirm that $V-M$ analysis provides an accurate account of inhibitory neurotransmission in this system. Mean $P_{\mathrm{r}}$, provided by $V-M$ analysis, shows a dependence on extracellular $\mathrm{Ca}^{2+}$ concentration that is nearly identical for both excitatory and inhibitory autapses. Finally, the $V-M$ method allows us to compare the locus of short-term synaptic plasticity at these connections. Glutamatergic autapses exhibit paired-pulse depression that depends mainly on changes in $P_{\mathrm{r}}$, whereas depression at GABAergic autapses appears to depend primarily on changes in the number of release sites. We conclude that, apart from differences in the mechanisms of short-term plasticity, the basic quantal properties of excitatory and inhibitory connections in this hippocampal system are remarkably similar.
\end{abstract}

Key words: autapse; calcium; EPSC; IPSC; probability; quantal analysis

\section{Introduction}

The dynamic interplay between synaptic excitation and inhibition is critical for normal information processing in the CNS. This interaction is ultimately constrained by the properties of individual excitatory and inhibitory synapses. Hence, an understanding of the dynamics of excitation and inhibition in the brain needs to be built upon an understanding of synaptic biophysics, for which the dominant paradigm remains the quantal theory of synaptic transmission. The quantal theory describes neurotransmission in terms of three parameters, N, $P_{\mathrm{r}}$, and Q (Del Castillo and Katz, 1954; Redman, 1990). Here, $N$ is the number of presynaptic sites at which synaptic vesicles are released, $P_{\mathrm{r}}$ is the probability that a single vesicle of neurotransmitter is released from each release site following stimulation, and $Q$ is the amplitude of the postsynaptic electrical response elicited by the contents of one vesicle of neu-

Received July 17, 2008; revised 0ct. 28, 2008; accepted Nov. 3, 2008.

This work was supported by a PhD scholarship from The Australian National University to K.I. and by recurrent funding from the John Curtin School of Medical Research to J.M.B. We thank John Clements and Angus Silver for comments on this manuscript.

Correspondence should be addressed to Dr. John M. Bekkers, Division of Neuroscience, John Curtin School of Medical Research, The Australian National University, Canberra, Australian Capital Territory 0200, Australia. E-mail: john.bekkers@anu.edu.au.

D0I:10.1523/JNEUROSCI.3350-08.2008

Copyright $\odot 2008$ Society for Neuroscience ～0270-6474/08/2813563-11\$15.00/0 rotransmitter. Although the quantal model is now part of the neuroscience vernacular, its details continue to be debated (Christie and Jahr, 2006; Ninio, 2007).

Recently, a new method of extracting the quantal parameters of synapses has been described, the variance-mean $(V-M)$ technique (also called multiprobability fluctuation analysis; Silver et al., 1998; Reid and Clements, 1999; Clements and Silver, 2000). This technique is appealing because, in its simplest form, it is easier to apply than the traditional, histogram-based quantal analysis (Stricker and Redman, 2003). Thus, the $V-M$ approach has increasingly been used to assess functional changes at synapses (Oleskevich et al., 2000; Foster and Regehr, 2004; Sargent et al., 2005; Biró et al., 2006; Zhang et al., 2006; Baldelli et al., 2007). However, despite its growing popularity, the $V-M$ technique has not yet been empirically verified in a system that previously has been well characterized using other methods of quantal analysis.

A second characteristic of the $V-M$ approach is that, to date, it has been applied largely to excitatory, glutamate-releasing synapses (e.g., Biró et al., 2005; Sargent et al., 2005; Tyler et al., 2006) (cf. Biró et al., 2006). Previous work, using various techniques, has indicated that synapses releasing the inhibitory neurotransmitter GABA cannot simply be assumed to exhibit quantal properties identical to those of excitatory synapses (Fedulova et al., 1999; Gitler et al., 2004; Sakaba, 2008). Hence, it will be helpful to use the $V-M$ technique to make a careful comparison between 
excitatory and inhibitory synapses under identical recording conditions.

In this paper we apply the $V-M$ technique to hippocampal autaptic cultures. First, we validate the $V-M$ approach for excitatory autaptic currents, testing in particular its sensitivity to the known heterogeneity in $P_{\mathrm{r}}$ and $Q$, which have been measured in culture using alternative methods (Rosenmund et al., 1993; Liu et al., 1999). Second, we apply the same approach to inhibitory autaptic currents. We find that $V-M$ analysis is a robust approach that is weakly sensitive to inhomogeneities in neurotransmission. Furthermore, our analysis reveals that excitatory and inhibitory neurotransmission exhibit near-identical sensitivity to extracellular calcium concentrations, but differ in their mechanisms of short-term plasticity.

\section{Materials and Methods}

Cell culture

Cultures were prepared from newborn Wistar rats or GAD67-GFP $(\Delta$ neo $)$ mice. Here we will refer to GAD67-GFP $(\Delta$ neo $)$ mice as GAD67GFP mice. These mice express GFP in GABA-containing neurons, facilitating their identification (Tamamaki et al., 2003). Animals were rapidly decapitated according to methods approved by the Animal Experimentation Ethics Committee of The Australian National University. The CA1 region of the hippocampus was dissected out, dissociated in a papain enzyme solution, and plated at a density of $8-10 \times 10^{4}$ cells $/ \mathrm{ml}$ on 12-mm-diameter round glass coverslips coated with agarose and spotted with a mixture of calf skin collagen $(0.5 \mathrm{mg} / \mathrm{ml}$; Contact Technologies) and poly-D-lysine $(0.1 \mathrm{mg} / \mathrm{ml}$; Sigma-Aldrich) using a glass microatomizer (fabricated in-house). For the mouse cultures, neurons were plated on coverslips with a monolayer of glial cells. The culture medium contained (per $100 \mathrm{ml}$ ): $50 \mathrm{ml}$ of DMEM with bicarbonate (D-0422; SigmaAldrich), $50 \mathrm{ml}$ of DMEM with HEPES (D-1152; Sigma-Aldrich), $2 \mathrm{ml}$ of B-27 serum supplement (Invitrogen), $5 \mathrm{ml}$ of fetal bovine serum (Millipore Bioscience Research Reagents), and $1 \mathrm{ml}$ of penicillin/streptomycin ( $100 \times$; Invitrogen), $\mathrm{pH} 7.6$ in a $5 \% \mathrm{CO}_{2}$ incubator at $37^{\circ} \mathrm{C}$. Five to seven days following plating, cytosine- $\beta$-D-arabinofuranoside ( $5 \mu \mathrm{m}$ final concentration; Sigma-Aldrich) was added to prevent further proliferation of glial cells. Cultures were fed once a week with culture medium containing D-(-)-2-amino-5-phosphonovaleric acid (D-APV, $20 \mu \mathrm{M}$; Tocris) to minimize excitotoxicity.

\section{Electrophysiology}

Neurons were used for recordings after 11-18 d in vitro. To record autaptic EPSCs, we selected a single, isolated rat or GFP-negative mouse neuron on a microisland (diameter $\sim 50-200 \mu \mathrm{m}$ ). In the case of rat cultures, nearly every neuron selected in this way was excitatory, because of the predominance of pyramidal neurons in region CA1. This identification was confirmed by blockade of the synaptic current by 6-cyano-7nitroquinoxaline-2,3-dione (CNQX, $20 \mu \mathrm{M}$; Tocris) and by its rapid ( $\sim 5$ ms) decay time constant (Bekkers, 2005). To record autaptic IPSCs, we selected a GFP-positive mouse neuron on a microisland that was not occupied by any other GFP-positive neurons. GFP-negative (presumed excitatory) neurons were typically also present on the island because of the much greater prevalence of excitatory neurons in these cultures. EPSC contamination from these neurons was blocked by the addition of CNQX $(20 \mu \mathrm{M})$ to all external solutions. The identification of autaptic IPSCs was also confirmed by blockade of the current by bicuculline (10 $\mu \mathrm{M}$; Tocris) and by its slower ( $\sim 50 \mathrm{~ms})$ weighted decay time constant (Baldelli et al., 2005).

Whole-cell patch-clamp recordings were obtained using a Multiclamp 700A amplifier (Molecular Devices). Patch electrodes had resistances of 2-3.5 $\mathrm{M} \Omega$ when filled with internal solution, which had the following composition (mM): $135 \mathrm{KCl}, 7 \mathrm{NaCl}, 1$ EGTA, $2 \mathrm{MgCl}_{2}, 2 \mathrm{Na}_{2} \mathrm{ATP}, 0.3$ NaGTP, 20 phosphocreatine, 10 HEPES, adjusted to pH 7.2 with $\mathrm{KOH}$, $310 \mathrm{mOsm} / \mathrm{kg}$. Input resistance and series resistance were continuously monitored using a $10 \mathrm{mV}$ hyperpolarizing voltage-clamp step. Series resistance compensation was adjusted to $65-85 \%$ at a bandwidth of 10 $\mathrm{kHz}$. Recordings were discarded if the series resistance varied by $>10 \%$ over the course of the experiment. Neurons were voltage clamped at -60 $\mathrm{mV}$, and pairs of autaptic currents (interpulse interval $50 \mathrm{~ms}$ for EPSCs, $350 \mathrm{~ms}$ for IPSCs) were elicited with a 2-ms-long step depolarization to 0 $\mathrm{mV}$ at 5 or $10 \mathrm{~s}$ intervals. The different intervals between paired stimuli for EPSCs and IPSCs were chosen because they are short enough to reveal strong short-term plasticity, but not so short that temporal overlap of postsynaptic currents occurs. (IPSCs decay much more slowly than EPSCs; see above.) Miniature EPSCs or IPSCs were recorded during the $5-10 \mathrm{~s}$ pauses between paired stimuli. Current was filtered at $10 \mathrm{kHz}$ using a 4-pole Bessel filter and digitized at $50 \mathrm{kHz}$ (Instrutech ITC-18; Instrutech/HEKA). Stimulation and data acquisition were controlled by AxoGraph 4.9 or AxoGraph X (AxoGraph Scientific). The bath solutions contained the following (in $\mathrm{mM}$ ): $125 \mathrm{NaCl}, 3 \mathrm{KCl}, x \mathrm{CaCl}_{2}, y \mathrm{MgCl}_{2}, 10$ glucose, 25 HEPES, adjusted to $\mathrm{pH} 7.4$ with $\mathrm{NaOH}, 25$ sorbitol (310 $\mathrm{mOsm} / \mathrm{kg}$ ), where $x$ and $y$ were changed to vary the release probability, with $x+y=11$ mм. For example, the solution labeled "2 Ca" (see Fig. 2) contains $2 \mathrm{~mm} \mathrm{CaCl}_{2}$ and $9 \mathrm{~mm} \mathrm{MgCl}_{2}$. An array of 8 flowpipes (each 500 $\mu \mathrm{m}$ internal diameter) was used to continuously perfuse an isolated neuron with external solution. Because the diameter of the flowpipes was much larger than that of the microisland, all autapses were uniformly bathed in the solution of choice. Solution exchange was achieved within $1 \mathrm{~s}$ by lateral movement of the flowpipes between autaptic stimuli. To assess the firing properties of GFP-positive interneurons, current clamp recordings were made from cells held near $-60 \mathrm{mV}$ by current injection, then a series of 200-ms-long current steps was injected. In these experiments, bridge balance and capacitance neutralization were carefully adjusted. TPMPA [(1,2,5,6-tetrahydropyridin-4-yl) methylphosphonic acid], CGP 55845, and gabazine (SR 95531) were obtained from Tocris. All experiments were performed at room temperature $\left(21-25^{\circ} \mathrm{C}\right)$.

\section{Immunohistochemistry}

Cultures were fixed for $20 \mathrm{~min}$ in $4 \%$ paraformaldehyde in phosphate buffer, then washed and stored in PBS. Cells were permeabilized with $0.3 \%$ Triton plus $1 \%$ bovine serum albumin in PBS for $1 \mathrm{~h}$, washed, then incubated overnight at $4^{\circ} \mathrm{C}$ in primary antibody. The antibodies and dilutions were: rabbit anti-GABA (Sigma-Aldrich, 1:2000); rabbit antisynapsin (gift from P. V. de Camilli, Yale University, New Haven, CT, 1:100). Following a further wash, cells were incubated for $4 \mathrm{~h}$ at $4^{\circ} \mathrm{C}$ in secondary antibody (Alexa 633 goat anti-rabbit IgG, 1:1000; Invitrogen). Fluorescence images were recorded using a Zeiss Pascal confocal microscope (Carl Zeiss).

\section{Variance-mean theory}

$V-M$ theory assumes that (1) a synaptic connection consists of a fixed population of $N$ release sites, each of which generates a unitary response with amplitude $Q$ and a fixed probability $P_{\mathrm{r}}$ following stimulation; and (2) the $Q$ values sum linearly to produce a net synaptic current $I$. These assumptions are similar to those of conventional quantal analysis (Del Castillo and Katz, 1954; Redman, 1990). However, the $V-M$ approach differs in the way in which experiments are performed and analyzed. A typical $V-M$ experiment involves recording 50-200 synaptic currents (fewer than required for conventional quantal analysis; Stricker and Redman, 2003), then repeating these recordings in a range of 4-8 different bath solutions, each of them producing a different fixed $P_{\mathrm{r}}$ (e.g., the solutions may contain different $\mathrm{Ca}$ concentrations). In each bath solution, the average synaptic current, $I$, and the variance of this current, $\sigma^{2}$, are given by the following (Clements and Silver, 2000):

$$
\begin{gathered}
I=N Q P_{\mathrm{r}} \\
\sigma^{2}=N Q^{2} P_{\mathrm{r}}\left(1-P_{\mathrm{r}}\right) .
\end{gathered}
$$

Eliminating $P_{\mathrm{r}}$ between these two equations gives the following:

$$
\sigma^{2}=Q I-I^{2} / N+\sigma_{\text {noise }}^{2}
$$

where the last term, empirically added, represents baseline variance due to instrument noise, etc. Analysis involves plotting $\sigma^{2}$ versus I measured in each of the bath solutions and fitting a parabola (Eq. 3) to estimate values for the two free parameters, $Q$ and $N$. This analysis, here called the "simple" analysis, also works if $Q$ and $P_{\mathrm{r}}$ are different across release sites 
A
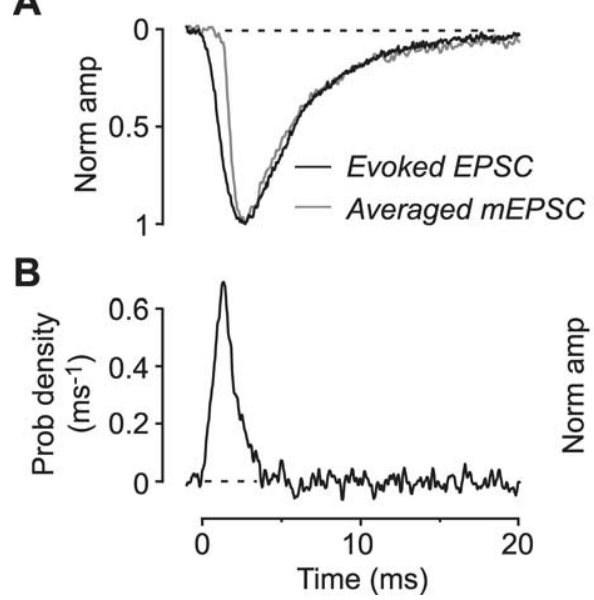

C

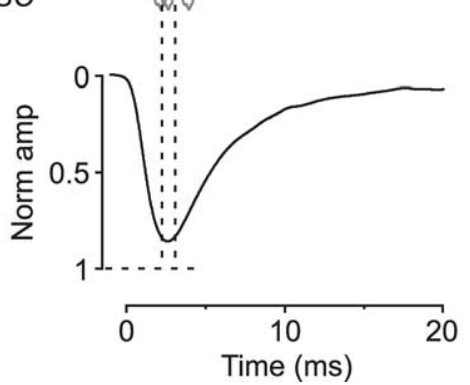

Figure 1. Variance due to jitter in the release of synaptic vesicles can be estimated using simulations. $\boldsymbol{A}$, Black trace is the normalized average ( $n=58)$ of autaptic EPSCS evoked in $2 \mathrm{~mm} \mathrm{Ca}^{2+}$, after subtracting the average $(n=22)$ of stimulus transients recorded in the same cell in $20 \mu \mathrm{m}$ CNQX. Gray trace is the normalized average $(n=194)$ of miniature EPSCs (mEPSCs) recorded in this cell in $0.8 \mathrm{~mm} \mathrm{Ca}^{2+}$. The slower rise time of the evoked EPSC is due to release jitter. $\boldsymbol{B}$, Numerical deconvolution of the mEPSC time course from the evoked EPSC time course $(\boldsymbol{A})$, giving the distribution of latencies to release of synaptic vesicles. C, Multiple $(n=450)$ copies of the normalized average mEPSC were overlaid after displacing them in time with the distribution of latencies shown in $\boldsymbol{B}$. Top panel shows three of these displaced mEPSCs; lower panel shows the average of all $450 \mathrm{mEPSC}$. This average has the same time course as the evoked EPSC $(\boldsymbol{A})$ but a reduced amplitude $(0.86)$ because of asynchronous summation of the displaced $\mathrm{mEPSC}$. Dashed lines represent the time window (peak of average $\pm 0.5 \mathrm{~ms}$ ) over which the amplitudes of the 450 displaced mEPSCs were measured (top) to estimate the variance due to release jitter (see Materials and Methods).

amounts of neurotransmitter released from each vesicle (Bekkers et al., 1990) or postsynaptic channel noise (Singer and Berger, 1999); and (2) variance due to jitter in the latency of release of vesicles $\left(\mathrm{CV}_{\mathrm{I}, j}^{2}\right)$ (Silver, 2003). Variance from source 1 has been directly measured in hippocampal cultures using a loose-patch method to record from a single excitatory synapse (Forti et al., 1997). These experiments gave $\mathrm{CV}_{\mathrm{I}, \mathrm{qa}}^{2} \sim$ 0.09. Loose-patch experiments have not been reported for inhibitory synapses, although single-bouton stimulation experiments have suggested that $\mathrm{CV}_{\mathrm{I}, \mathrm{qa}}^{2} \sim 0.025-0.061$ for unitary IPSCs in hippocampal cultures (Fedulova et al., 1999). Because this estimate is relatively inaccurate, we decided to use the larger value from excitatory synapses (0.09) for our inhibitory analysis to avoid underestimating the importance of this parameter for our fits.

$\mathrm{CV}_{\mathrm{I}, j}^{2}$ was estimated for each cell using simulations. First, the transmitter release time course was calculated as follows. Miniature postsynaptic currents (mPSCs, either excitatory or inhibitory) were captured using the template scaling algorithm in AxoGraph X (AxoGraph Scientific). Because each cell is physically isolated from its neighbors, all active synapses on that cell arise from itself; hence, both spontaneous mPSCs and action potential-evoked PSCs arise from the same population of autapses.

(but are constant at any one site). In this case the $Q$ obtained from the fit to Equation 3 is an average across sites, weighted by the value of $P_{\mathrm{r}}$ at each site (Clements and Silver, 2000).

Although the simple analysis incorporates baseline noise variance, it does not explicitly include additional biological variance. This arises from three main sources: (1) intrasite (type I) variance, due to trial-totrial fluctuations in both the size and latency of the unitary response at each release site; (2) intersite (type II) variance, due to differences in the size of the mean unitary response between different sites; and (3) nonuniform $P_{\mathrm{r}}$ between different sites. Intrasite and intersite variance can be quantified as $\mathrm{CV}_{\mathrm{I}}^{2}$ and $\mathrm{CV}_{\mathrm{II}}^{2}$, respectively, where the squared coefficient of variation $\left(\mathrm{CV}^{2}\right)$ of a fluctuating quantity is defined as its variance divided by the square of its mean. Our methods of estimating $\mathrm{CV}_{\mathrm{I}}^{2}$ and $\mathrm{CV}_{\mathrm{II}}^{2}$ are described below.

Correction for the third factor, nonuniform $P_{\mathrm{r}}$, requires knowledge of the distribution of $P_{\mathrm{r}}$ across release sites. Silver and colleagues (Silver et al., 1998; Silver, 2003; Saviane and Silver, 2006) have developed an analysis that assumes the distribution of $P_{\mathrm{r}}$ is described by families of beta functions. With this approach, the $P_{\mathrm{r}}$ distribution can be quantified using two parameters: the mean $P_{\mathrm{r}}$ across all sites (which can be obtained from Eq. 1 above), and a shape parameter, $\alpha$. Our method of estimating $\alpha$ is described below.

Incorporating these three corrections, the $V-M$ equation becomes as follows (Silver et al., 1998):

$$
\sigma^{2}=\left[Q I-\frac{Q I^{2}(1+\alpha)}{I+N Q \alpha}\right]\left(1+C V_{I I}^{2}\right)+\mathrm{Q} I C V_{I}^{2}+\sigma_{\text {noise }}^{2} .
$$

Analysis using this equation, here called the "complex" analysis, involves first measuring (fixed) values for $\mathrm{CV}_{\mathrm{I}}^{2}, \mathrm{CV}_{\mathrm{II}}^{2}, \alpha$, and $\sigma_{\text {noise }}^{2}$, then fitting Equation 4 to $V-M$ data to estimate the two free parameters, $Q$ and $N$.

Note that another variant of $V-M$ analysis, intermediate between Equations 3 and 4 , involves setting $\mathrm{CV}_{\mathrm{II}}^{2}=0$ (no intersite variance) and $\alpha \rightarrow \infty$ (uniform $P_{\mathrm{r}}$ ) (Reid and Clements, 1999; Clements and Silver, 2000; Oleskevich et al., 2000). This version of the theory was not explicitly tested here.

\section{Estimation of $C V_{I}^{2}, C V_{I I}^{2}$, and $\alpha$}

Intrasite variance, $C V_{I}^{2}$. This has two main sources: (1) variance due to fluctuations in quantal amplitude $\left(\mathrm{CV}_{\mathrm{I}, \mathrm{qa}}^{2}\right)$, perhaps due to variable
Captured mPSCs were optimally aligned by the detection algorithm, then averaged ( $n=64-569$ events per cell); this provides the time course of synaptic current in the absence of release jitter (Fig. $1 \mathrm{~A}$, gray trace). Evoked PSCs, recorded in the same cell in external solution containing $0.8-2 \mathrm{~mm} \mathrm{Ca}^{2+}$, were averaged $(n=30-58)$. Use of smaller PSCs recorded in lower $\mathrm{Ca}^{2+}$ concentrations minimized nonlinear interactions between quanta that might compromise the analysis (Neher and Sakaba, 2001). Stimulus transients were removed by subtracting averaged currents $(n=12-30)$ recorded in the cell following addition of $20 \mu \mathrm{M}$ CNQX or $10 \mu \mathrm{M}$ bicuculline to block EPSCs or IPSCs, respectively. Averaged evoked PSCs (Fig. 1 A, black trace) had slower kinetics than averaged mPSCs (Fig. $1 A$, gray trace), reflecting asynchronous release of quanta of neurotransmitter. The release time course was calculated for each cell by deconvolving the normalized average MPSC from the normalized average PSC using a Fourier transform approach with a Wiener filter (Fig. $1 B)$ (Diamond and Jahr, 1995). This release time course had a mean half-width of $2.4 \pm 0.7 \mathrm{~ms}(n=7$ cells $)$ for EPSCs and $1.6 \pm 0.4 \mathrm{~ms}(n=$ 6) for IPSCs. Next, multiple $(n=400-1500)$ instances of the normalized average $\mathrm{MPSC}$ were generated with a distribution of latencies described by the deconvolved release time course (Fig. $1 C$, top panel). The average of these mPSCs reproduced the slower time course of the original normalized PSC, as expected, but its amplitude was smaller than unity because of the temporally dispersed summation of mPSCs (Fig. 1C, bottom panel). This amplitude difference, here called the "jitter amplitude scale factor," was used to correct the estimate of $Q$ obtained from $V-M$ analysis (see Results). The peak time of this average of jittery mPSCs, $t_{\text {peak }}$, was noted, then the average amplitude within the fixed time window, $t_{\text {peak }} \pm$ $0.5 \mathrm{~ms}$, was measured for each of these jittery mPSCs. Sometimes the measurement window straddled the peak of the mPSC; at other times it fell on the rising or falling phase (Fig. 1C, top panel). Because the mPSCs had normalized amplitudes, the variability in these amplitude measurements could be equated to the variance due to jitter in the release of synaptic vesicles, that is, $\mathrm{CV}_{\mathrm{I}, j}^{2}$. Finally, the total intrasite variance for each cell was calculated as $\mathrm{CV}_{\mathrm{I}}^{2}=\mathrm{CV}_{\mathrm{I}, \mathrm{qa}}^{2}+\mathrm{CV}_{\mathrm{I}, j}^{2}$.

Intersite variance, $C V_{I I}^{2}$. This arises from differences in the mean amplitude of unitary postsynaptic events between release sites. The amplitude variance of all mPSCs recorded in the whole cell, $\mathrm{CV}_{\mathrm{wc}}^{2}$, is equal to the sum of $\mathrm{CV}_{\mathrm{II}}^{2}$ and the above-mentioned single-site variance, $\mathrm{CV}_{\mathrm{I}, \mathrm{qa}}^{2}$ $\left(\sim 0.09\right.$ at excitatory synapses). Hence, after measuring $\mathrm{CV}_{\mathrm{wc}}^{2}, \mathrm{CV}_{\mathrm{II}}^{2}$ can be 
estimated by subtraction. $\mathrm{CV}_{\mathrm{wc}}^{2}$ was measured in a separate series of experiments optimized for the detection of MPSCs (i.e., with precautions to minimize recording noise). Spontaneous mPSCs were captured using the detection algorithm in AxoGraph $\mathrm{X}$, and their amplitudes were measured by averaging over a fixed time window, $t_{\text {peak }} \pm 0.5 \mathrm{~ms}$, where $t_{\text {peak }}$ is the peak of the averaged captured mPSCs. For excitatory autapses, mean $\mathrm{CV}_{\mathrm{wc}}^{2}=0.285 \pm 0.040(n=6)$, which is similar to the value reported for excitatory hippocampal synapses in culture $(0.303 \pm 0.017$; Bekkers et al., 1990). Subtracting the single-site variance (0.09) yields $\mathrm{CV}_{\mathrm{II}}^{2}=0.195$ for excitatory autapses. For inhibitory autapses, mean $\mathrm{CV}_{\mathrm{wc}}^{2}$ $=0.237 \pm 0.035(n=7)$, giving $\mathrm{CV}_{\mathrm{II}}^{2}=0.147$ (assuming the same single-site variance as for excitatory synapses).

Beta function parameter, $\alpha$, describing the distribution of $\mathrm{P}_{r}$. The distribution of $P_{\mathrm{r}}$ for excitatory synapses in hippocampal cultures has been experimentally determined using an optical approach (Murthy et al., 1997). Those authors fitted their data to an alpha function. We refitted their data to the beta function used here, giving $\alpha \sim 1.7$ (see also Clements and Silver, 2000). Similar experiments have not been done for inhibitory synapses, and so the same value of $\alpha$ was assumed. We examined the validity of this assumption by refitting Equation 4 to the inhibitory data while setting $\alpha$ to a wide range of different values (supplemental material, available at www.jneurosci.org). This showed that, to maintain acceptable fits of Equation 4 to the $V-M$ data for inhibitory synapses, the fitted values of $Q$ and $N$ cannot differ by more than approximately $\pm 12 \%$ and $\pm 20 \%$, respectively, from the values estimated on the assumption that $\alpha=1.7$.

\section{Data analysis}

Autaptic PSCs recorded in the same extracellular $\mathrm{Ca}^{2+}$ concentration $\left(\left[\mathrm{Ca}^{2+}\right]_{\mathrm{e}}\right)$ were averaged together and $t_{\text {peak }}$ of this average was noted. Episodes were adjusted to the baseline immediately before the stimulus step, then individual PSC amplitudes were measured by averaging over the window $t_{\text {peak }} \pm 0.5 \mathrm{~ms}$, using the $t_{\text {peak }}$ appropriate to that $\left[\mathrm{Ca}^{2+}\right]_{\mathrm{e}}$. Background noise was measured by using a similar procedure, using the average of all $t_{\text {peak }}$ values, following blockade of EPSCs or IPSCs with CNQX or bicuculline, respectively. This measured noise variance was explicitly included in the fits to Equations 3 and 4, but in every case it was negligible. A plot of PSC amplitude versus time (e.g., see Fig. $2 B$ ) was examined by eye for stability. Amplitudes that transiently diverged from the average (e.g., the first one or two points after a solution change) (see Figs. $2 B, 4 B$ ) were excluded from analysis. Plots of input resistance and the peak amplitude of the sodium current elicited by the 2-ms-long depolarizing step stimulus were also checked for stability (see Fig. $2 B$ ). The latter is very sensitive to changes in the excitability of the cell, as well as to changes in series resistance. For some cells and bath solutions, the effect of slow changes in PSC amplitude was minimized by calculating both the mean amplitude and the sample variance of the amplitude from groups of 5 or 10 consecutive measurements. These averages were then averaged together to produce estimates of $I$ and $\sigma^{2}$ for each $\left[\mathrm{Ca}^{2+}\right]_{\mathrm{e}} \cdot \mathrm{CV}_{\mathrm{I}}^{2}$ was estimated for each cell, as described above. $\mathrm{CV}_{\mathrm{II}}^{2}$ and $\alpha$ were assigned fixed values obtained from averages across cells (above). A plot of $\sigma^{2}$ versus I for each cell was fitted to either the simple or complex theory (Eq. 3 or 4 , respectively). The errors in the variance ( \pm SD, error bars in Figs. $2 C, 4 C$ ) were estimated using a bootstrap procedure, as follows. For each list of $n$ PSC amplitudes, a new list was generated by randomly resampling $n$ times, with replacement, and $\sigma^{2}$ was calculated for this new list. The resampling procedure was repeated 1000 times, then the SD of all 1000 values of $\sigma^{2}$ was calculated. To generate the plot of $P_{\mathrm{r}}$ versus $\left[\mathrm{Ca}^{2+}\right]_{\mathrm{e}}$ (see Fig. 5), the fitted values of $N$ and $Q$ were used in Equation 1 to calculate $P_{\mathrm{r}}$, knowing $I$ for each $\left[\mathrm{Ca}^{2+}\right]_{\mathrm{e}}$. Values of $P_{\mathrm{r}}$ for the same $\left[\mathrm{Ca}^{2+}\right]_{\mathrm{e}}$ in different cells were then averaged together. Data points in Figure 5 were fitted to the Hill equation:

$$
y=a /\left[1+\left(c_{1 / 2} / \mathrm{x}\right)^{\mathrm{h}}\right]
$$

where $a$ is the asymptotic maximal amplitude, $c_{1 / 2}$ is the half-maximal $\mathrm{Ca}^{2+}$ concentration, $x$ is $\left[\mathrm{Ca}^{2+}\right]_{\mathrm{e}}$, and $h$ is the slope or Hill coefficient. All analysis was done using AxoGraph X or Igor Pro (Wavemetrics). Curve fitting used the nonlinear least-squares Levenberg-Marquardt algorithm implemented in Igor Pro. Unless stated otherwise, errors are given as $\pm \mathrm{SE}$ and statistical comparisons were made using the two-tailed paired or unpaired $t$ test with significance at $p=0.05$ and $n$ equal to the number of cells.

\section{Simulations}

The effect on $V-M$ plots of $P_{\mathrm{r}}$-dependent changes in $Q$ and $N$ (see Fig. $7 A$ ) was simulated as follows. $Q$ (and $N$ ) was modeled as varying with $P_{\mathrm{r}}$ according to the following:

$$
Q=0.2+\left(1-P_{\mathrm{r}}^{4}\right) / 1.25
$$

This causes $Q$ ( or $N$ ) to be 1 at low $P_{\mathrm{r}}$, rolling off to 0.2 at high $P_{\mathrm{r}}$ (see Fig. $7 A$, inset). For each value of $P_{\mathrm{r}}(0-0.8$ in steps of 0.01$), Q$ (or $\left.N\right)$ was calculated from Equation 6 and then substituted into Equations 1 and 2 to find $I$ and $\sigma^{2}$. Finally, $\sigma^{2}$ was plotted against $I$ for each $P_{\mathrm{r}}$, giving the distorted parabolas in Figure $7 \mathrm{~A}$.

\section{Results \\ Autaptic EPSCs exhibit amplitude fluctuations that vary with $P_{\mathrm{r}}$}

We began by assessing the validity of the $V-M$ approach for autaptic EPSCs in single, isolated pyramidal neurons in cultures prepared from area CA1 of the hippocampus. This is a well defined system with a number of important advantages: (1) the single-site quantal variance (Forti et al., 1997) and distribution of release probabilities, $P_{\mathrm{r}}$ (Murthy et al., 1997; Liu et al., 1999), are known, making it possible to test a more rigorous version of the $V-M$ method (Eq. 4) (Silver, 2003); (2) all synaptic contacts on the cell arise from an axon emanating from the cell itself, meaning that all spontaneous and evoked EPSCs must originate from the same defined population of autapses; and (3) $\left[\mathrm{Ca}^{2+}\right]_{\mathrm{e}}$ can be varied over a wide range (as a means of widely varying $P_{\mathrm{r}}$ ) without risk of polysynaptic contamination, given that all autapses onto an isolated neuron are necessarily monosynaptic.

The identity of autaptic EPSCs, elicited using a paired-pulse protocol and recorded under whole-cell voltage clamp, was confirmed by their pharmacology and kinetics (Fig. 2A) (Materials and Methods). Average $P_{\mathrm{r}}$ was varied by stepping $\left[\mathrm{Ca}^{2+}\right]_{\mathrm{e}}$ to a range of values $(0.8,1.2$ or $1.5,2,5 \mathrm{~mm}$, and sometimes $10 \mathrm{~mm})$ (Fig. 2A). The total extracellular divalent cation $\left(\mathrm{Ca}^{2+}\right.$ plus $\mathrm{Mg}^{2+}$ ) concentration was usually kept constant (11 mM) to minimize perturbation of the axonal action potential (Frankenhaeuser and Hodgkin, 1957).

Unless stated otherwise, all measurements were made on the first of the paired autaptic currents. Following a step change in $\left[\mathrm{Ca}^{2+}\right]_{\mathrm{e}}$, the amplitude of the autaptic EPSC quickly settled to a constant mean value (Fig. $2 \mathrm{~B}$ ). The stability of recordings was verified by repeated application of the $2 \mathrm{mM} \mathrm{Ca}^{2+}$ solution, and by monitoring both the input resistance of the cell and the peak sodium current elicited during the depolarizing stimulus (Fig. $2 B)$. The mean amplitude of the autaptic EPSC increased with increasing $\left[\mathrm{Ca}^{2+}\right]_{\mathrm{e}}$. In contrast, fluctuations in the amplitude were smaller at low and high $P_{\mathrm{r}}$ and maximal in between (Fig. $2 B$ ). This behavior is expected of a fixed population of independent, stochastic synapses, as assumed by the $V-M$ theory (Clements and Silver, 2000).

\section{Fluctuations in autaptic EPSC amplitude are well fitted by both the "simple" and "complex" $V-M$ theories}

Amplitude fluctuations were quantified by calculating the sample variance $\left(\sigma^{2}\right)$ of the amplitude during stable recording epochs in a fixed $\left[\mathrm{Ca}^{2+}\right]_{\mathrm{e}}$ (indicated by the bars in Fig. $2 \mathrm{~B}$ ). A plot of $\sigma^{2}$ versus the mean EPSC amplitude $(I)$ under each $P_{\mathrm{r}}$ condition was well fitted by a parabola, as expected from the simple version of 

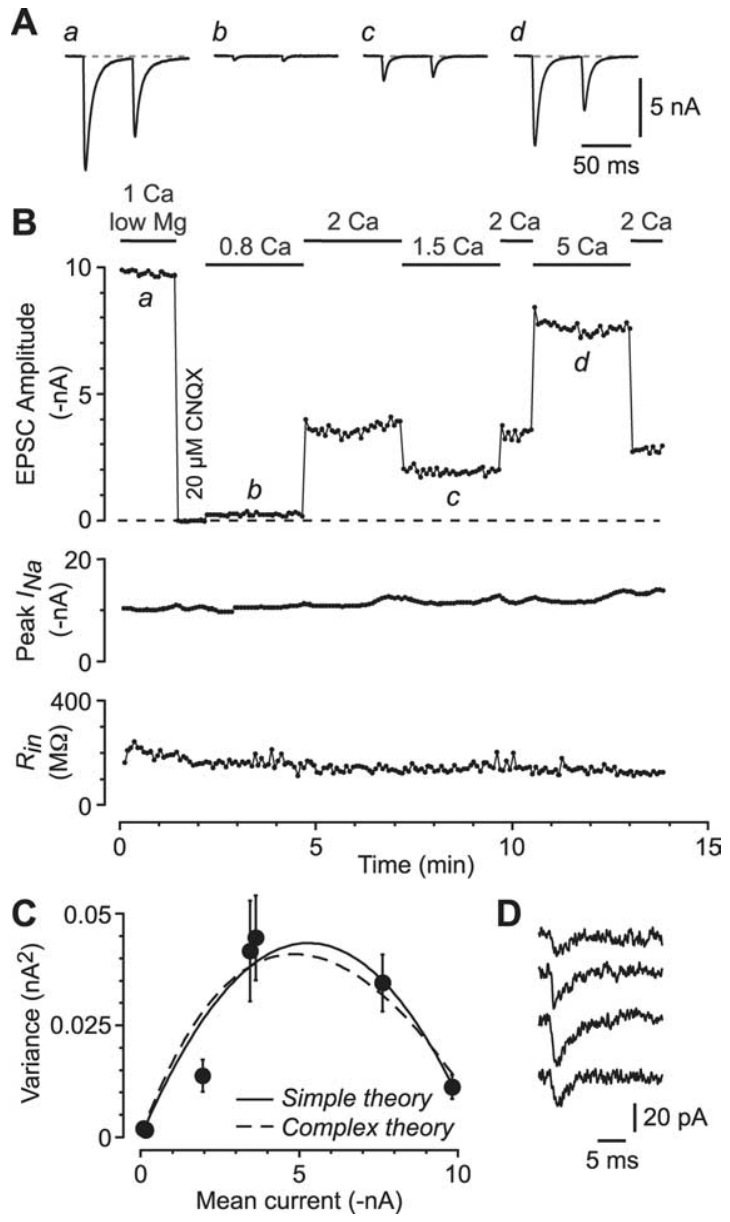

Figure 2. Amplitude and amplitude fluctuations of autaptic EPSCs vary with extracellular $\mathrm{Ca}^{2+}$ concentration $\left(\left[\mathrm{Ca}^{2+}\right]_{e}\right)$, and the data are well described by both the simple and complex versions of the $V-M$ theory. $\boldsymbol{A}$, Example autaptic EPSCs recorded at each of the time points $(\boldsymbol{a}-\boldsymbol{d})$ marked in $\boldsymbol{B}$. Pairs of stimuli (50 ms interval) were delivered; stimulus transients have been blanked. Same cell as in Figure 1. $\boldsymbol{B}$, Plots of the amplitude of the first EPSC in each pair (top panel), the peak sodium current during the stimulus (peak $I_{\mathrm{Na}}$; middle panel) and the input resistance of the cell ( $R_{\text {in }}$; bottom panel) versus time during the experiment. Periods of application of different $\left[\mathrm{Ca}^{2+}\right]_{e}$ solutions are indicated by the horizontal bars. Average EPSC amplitude is reasonably constant in any one solution; $I_{\mathrm{Na}}$ and $R_{\text {in }}$ are constant across all solutions. $\boldsymbol{C}$, Variance of EPSC amplitude fluctuations plotted against mean EPSC amplitude. Each point represents data obtained in a different $\left[\mathrm{Ca}^{2+}\right]_{\mathrm{e}}$ (same cell as in $\left.\boldsymbol{A}, \boldsymbol{B}\right)$. Error bars represent \pm 1 SD. Solid curve is a fit to the simple version of $V-M$ analysis (Eq. 3); dashed curve is a fit to the complex version (Eq. 4). D Typical mEPSCs recorded in the same cell.

$V-M$ analysis (Eq. 3) (Fig. 2C, superimposed solid curve). This fit yielded estimates for this cell of the average quantal amplitude $(\mathrm{Q}=16.3 \pm 1.7 \mathrm{pA}, \pm \mathrm{SD})$ and the number of release sites $(N=$ $648 \pm 85, \pm \mathrm{SD})$.

A more complex version of $V-M$ analysis takes into account the additional contributions of intersite and intrasite synaptic variance, as well as the nonuniform distribution of $P_{\mathrm{r}}$ across different release sites (Eq. 4) (Silver et al., 1998; Clements and Silver, 2000). These contributions are encapsulated in three additional parameters, $\mathrm{CV}_{\mathrm{I}}^{2}, \mathrm{CV}_{\mathrm{II}}^{2}$, and $\alpha$ (see Materials and Methods). $\mathrm{CV}_{\mathrm{II}}^{2}$ and $\alpha$ were obtained from population data (see Materials and Methods), whereas $C V_{\mathrm{I}}^{2}$ required measurement of the jitter in neurotransmitter release for each cell (Fig. 1; same cell as Fig. 2). For the cell shown in Figures 1 and $2, \mathrm{CV}_{\mathrm{I}}^{2}=0.13$ (compare average for $n=6$ cells, $\mathrm{CV}_{\mathrm{I}}^{2}=0.28 \pm 0.09$ ). These values were incorporated into the complex version of the $V-M$ equation (Eq. 4), which was then fitted to the data (Fig. $2 C$, superimposed dashed curve). Parameters obtained from the fit of Equation 4 $(Q=14.6 \pm 2.0 \mathrm{pA}, N=643 \pm 118, \pm \mathrm{SD})$ were very similar to the parameters obtained above from the fit of the simple parabola (Eq. 3).

Jitter in neurotransmitter release gives rise to an additional correction factor that must be applied to the fitted value of $Q$. This factor, the jitter amplitude scale factor (see Materials and Methods), corrects for the underestimate of $Q$ due to the temporally dispersed summation of unitary postsynaptic currents. It applies equally to the results of the simple and complex fits. For the cell in Figures 1 and 2, this scale factor is 1.17, i.e., the fitted value for $Q$ must be increased by this factor (compare average scale factor $1.49 \pm 0.17, n=7$ cells).

In summary, the mean values of $Q$ obtained from simple and complex fits to all pyramidal cells in our dataset were $43.1 \pm 11.6$ $\mathrm{pA}$ and $33.5 \pm 7.5 \mathrm{pA}$, respectively (after jitter amplitude correction), and the mean values of $N$ were $533 \pm 120$ and $602 \pm 174$, respectively (both pairs not significantly different, $p>0.05 ; n=$ 7 cells). Hence, the simple version of $V-M$ theory (Eq. 3 ) is weakly sensitive to heterogeneities in synaptic transmission in culture and provides an adequate description of our data.

\section{$V-M$ analysis of autaptic EPSCs correctly predicts quantal amplitude}

We next sought to validate $V-M$ analysis by comparing its estimate of $Q$ with the value obtained using an alternative approach. $Q$ is conventionally equated to the mean amplitude of spontaneous miniature synaptic currents arising from the same population of synapses as participate in the evoked response (Redman, 1990). In the case of an isolated neuron, all synapses on the cell participate, so $Q$ should correspond to the mean amplitude of all miniature synaptic currents recorded in that cell. Dendritic filtering, which will differentially distort the synaptic currents recorded at the soma (depending on the dendritic location of the active synapse), should identically affect the unitary synaptic currents whether they are measured in isolation (as miniatures) or as part of a compound evoked synaptic response (as from $V-M$ analysis). Miniature EPSCs were recorded from excitatory autapses (Fig. 2D) and compared with the jitter-corrected $Q$ obtained from simple $V-M$ analysis in the same cell. There was no significant difference between the two values $(Q / \mathrm{mEPSC}$ amplitude $=1.22 \pm 0.34, n=7, p>0.5$; average mEPSC amplitude $=$ $36.9 \pm 2.3 \mathrm{pA}, n=7$ cells). Thus, simple $V-M$ analysis correctly extracts the quantal amplitude, $Q$.

We also attempted to validate the $V-M$ estimate of $N$ by comparing it with the number of morphologically identified autaptic contacts on the same cell. Immunolabeling with a synapsin antibody was used to identify autapses. Unfortunately, the counts were unreliable because of the large number of overlapping labeled puncta on these densely self-innervated neurons. Qualitatively, this is in accordance with the large values of $N$ estimated from $V-M$ analysis $(\sim 500-600)$.

\section{GABAergic interneurons can reliably be identified in hippocampal cultures from GAD67-GFP transgenic mice} Having validated the $V-M$ method for excitatory autapses in hippocampal cultures, we next wished to apply this approach to cultured inhibitory autapses. Because GABAergic cells comprise only $\sim 10 \%$ of the neuronal population in the hippocampus (Spruston and McBain, 2007), and because excitatory and inhibitory neurons in hippocampal cultures are morphologically similar, it is difficult to find isolated autaptic GABAergic neurons by chance in culture. To overcome this problem, we prepared CA1 
cultures from newborn GAD67-GFP transgenic mice that specifically express GFP in GABAergic neurons under the control of the endogenous GAD67 promoter (Tamamaki et al., 2003). GFPpositive neurons can readily be targeted for recordings (Fig. 3A).

Every GFP-positive neuron in culture expressed GABA, as determined using immunohistochemistry (Fig. $3 B)(n=11$ cells). Furthermore, in every case where an autaptic current was recorded from a solitary GFP-positive neuron, the current exhibited the characteristics of a GABAergic IPSC: it was reversibly blocked by bath application of the $\mathrm{GABA}_{\mathrm{A}}$ receptor antagonist, bicuculline (10 $\mu \mathrm{M}$; data not shown), and its weighted decay time constant resembled that of IPSCs previously recorded in rat hippocampal cultures (mean = 49.2 $\pm 3.6 \mathrm{~ms}, n=10$; Baldelli et al., 2005).

GABAergic interneurons in the hippocampus are known to comprise a highly heterogeneous population (McBain and Fisahn, 2001). Accordingly, we tested for diversity in the patterns of action potential (AP) firing in response to a current step, as has been reported for hippocampal interneurons in situ (McBain and Fisahn, 2001). Two patterns of AP firing were observed in GFP-positive neurons (Fig. 3C). Regular-spiking cells exhibited a nonaccommodating train of APs during 200ms-long current steps ( $n=6$ cells) (Fig. $3 C$, right), whereas single-spiking cells showed only a single AP at the start of the step ( $n=4$ cells) (Fig. 3C, left). Hence, some degree of functional diversity of interneurons is preserved in culture.

\section{Autaptic IPSC amplitude fluctuations can be fitted to $V-M$ theory}

We recorded autaptic IPSCs from an isolated GFP-positive neuron on a microisland in a range of $\left[\mathrm{Ca}^{2+}\right]_{\mathrm{e}}$, as was done for autaptic EPSCs. As before, cell stability was confirmed by monitoring peak $\mathrm{Na}$ current and input resistance and by revisiting the $2 \mathrm{mM} \mathrm{Ca}^{2+}$ solution (Fig. $4 A, B$ ). A plot of $\sigma^{2}$ versus $I$ for these autaptic IPSCs was well fitted by the simple $V-M$ equation (Eq. $3)$, yielding $Q=17.4 \pm 3.2 \mathrm{pA}$ and $N=474 \pm 120$ for this cell (Fig. 4C, superimposed solid curve) ( $Q$ not corrected for jitter; $\pm \mathrm{SD}$ ). This plot was also reasonably fitted by the complex $V-M$ equation (Eq. 4), giving $Q=12.0 \pm 3.5 \mathrm{pA}$ and $N=390 \pm 183$ (Fig. 4C, superimposed dashed curve) ( $Q$ not corrected; $\pm S D$ ). Summarizing the results for $n=6$ inhibitory neurons, the mean values of $Q$ obtained from simple and complex fits were $23.1 \pm$ $3.0 \mathrm{pA}$ and $18.7 \pm 1.8 \mathrm{pA}$, respectively (after jitter amplitude correction), and the mean values of $N$ were $336 \pm 89$ and $301 \pm$ 64 , respectively (both pairs not significantly different, $p>0.05$ ). Hence, like autaptic EPSCs, $V-M$ data for autaptic IPSCs are adequately described by the simple version of the theory.

In an attempt to distinguish functional heterogeneity between different subtypes of interneurons, the simple $V-M$ analysis was done on regular-spiking $(n=3)$ and single-spiking $(n=5)$ cells (Fig. $3 C$ ). Although numbers are small, there was no significant difference between the results for the two cell types ( $p>0.5$; not illustrated).

As before, miniature postsynaptic currents were recorded from inhibitory autapses (Fig. 4D) and compared with the jittercorrected $Q$ obtained from simple $V-M$ analysis in the same cell. On average, $Q$ was $\sim 20 \%$ smaller than the mIPSC amplitude, perhaps because mIPSCs were often noisy and smaller events may have been missed ( $Q / \mathrm{mIPSC}$ amplitude $=0.79 \pm 0.06, n=6, p=$ 0.03 ; average mIPSC amplitude $=29.3 \pm 2.8 \mathrm{pA}, n=6$ ). Overall, however, there is again reasonable agreement between the two methods of estimating quantal amplitude.

\section{Cross talk between release sites occurs at GABAergic autapses in $10 \mathrm{~mm} \mathrm{Ca}{ }^{2+}$}

The $V-M$ approach assumes that changing $\left[\mathrm{Ca}^{2+}\right]_{\mathrm{e}}$ only changes $P_{\mathrm{r}}$, without having additional effects that might invalidate the method. Accordingly, we asked whether increasing $\left[\mathrm{Ca}^{2+}\right]_{\mathrm{e}}$ to 10 mu might cause cross talk between release sites (due to multivesicular release at one synapse, or spillover between synapses), possibly leading to contravention of the assumption that quanta summate linearly and independently (see Materials and Methods).

Cross talk between release sites, described by the authors as multivesicular release, has been reported at excitatory autapses in hippocampal cultures under high- $P_{\mathrm{r}}$ conditions (Tong and Jahr, 1994). Is the same effect apparent at inhibitory autapses? We compared the block of autaptic IPSCs by high- and low-affinity $\mathrm{GABA}_{\mathrm{A}}$ receptor competitive antagonists (gabazine and TPMPA, respectively) at 2 and $10 \mathrm{~mm} \mathrm{Ca}^{2+}$ in the same neuron (Hartman et al., 2006). Gabazine ( $80 \mathrm{~nm}$ ) blocked autaptic IPSCs equally in 


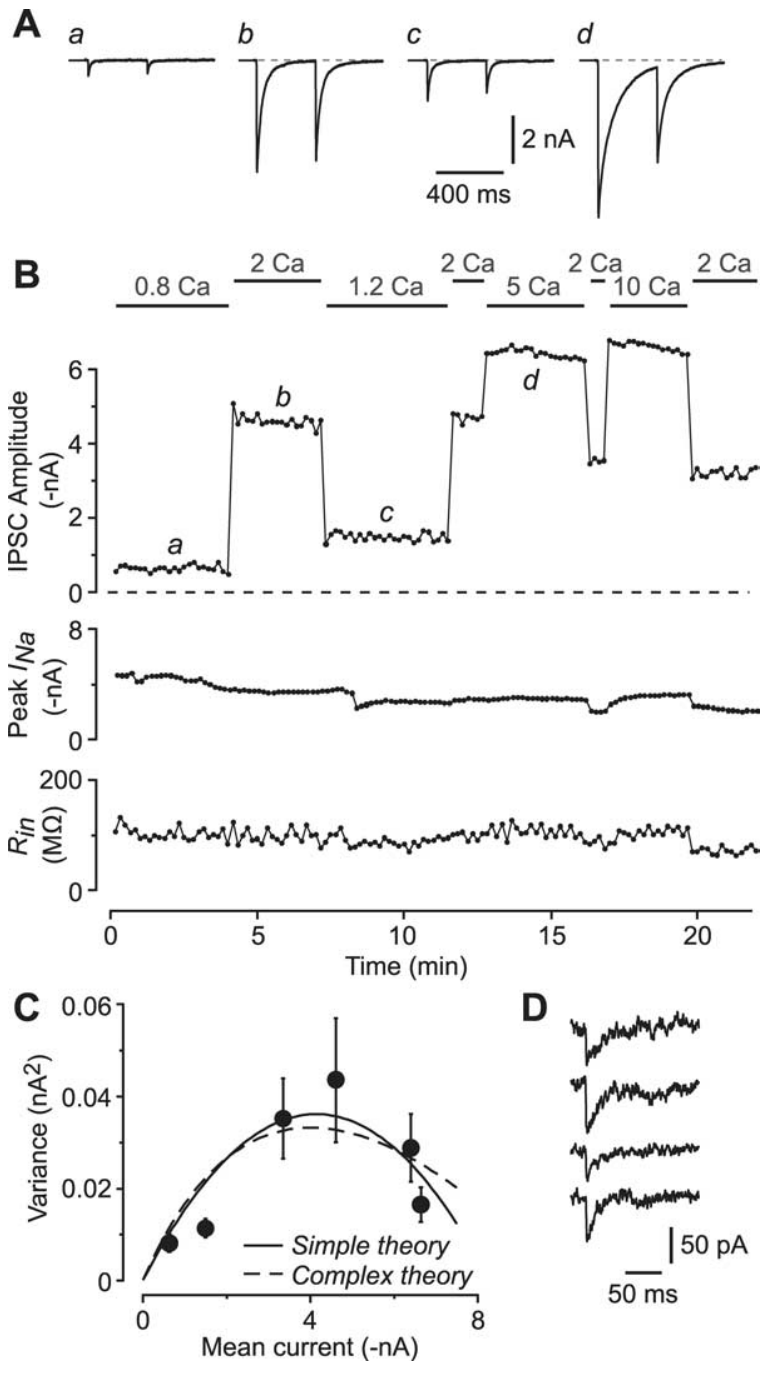

Figure 4. Amplitudes and amplitude fluctuations of autaptic IPSCs vary with $\left[\mathrm{Ca}^{2+}\right]_{e}$, and the data can be fitted to both the simple and complex versions of the $V-M$ theory. $A$, Illustrative autaptic IPSCs recorded at each of the time points $(\boldsymbol{a}-\boldsymbol{d})$ in $\boldsymbol{B}$. Pairs of stimuli were applied at a $350 \mathrm{~ms}$ interval. Stimulus transients have been blanked. $\boldsymbol{B}$, Plots of the amplitude of the first IPSC in each pair (top panel), peak $I_{\mathrm{Na}}$ during the stimulus (middle panel) and $R_{\text {in }}$ of the cell (bottom panel) versus time during the experiment. Periods of application of different $\left[\mathrm{Ca}^{2+}\right]_{e}$ solutions are indicated by the horizontal bars. C, Variance of IPSC amplitude fluctuations plotted against mean IPSC amplitude for each $\left[\mathrm{Ca}^{2+}\right]_{e}$ in $\boldsymbol{B}$. Error bars represent \pm 1 SD. Solid curve is a fit to Equation 3; dashed curve is a fit to Equation 4.D, Typical mIPSCs recorded in the same cell.

low and high $\mathrm{Ca}^{2+}$, as expected for a high-affinity competitive antagonist $\left(2 \mathrm{mM} \mathrm{Ca}^{2+}: 36.3 \pm 2.1 \%\right.$ block, $n=4 ; 10 \mathrm{mM} \mathrm{Ca}^{2+}$ : $35.0 \pm 1.9 \%$ block, $n=4 ; p=0.82)$. In contrast, TPMPA $(200$ $\mu \mathrm{M})$ blocked autaptic IPSCs less in $10 \mathrm{~mm}$ than in $2 \mathrm{mM} \mathrm{Ca}^{2+}(2$ $\mathrm{mM} \mathrm{Ca}^{2+}: 40.7 \pm 1.0 \%, n=4 ; 10 \mathrm{mM} \mathrm{Ca}^{2+}: 29.2 \pm 1.0 \%, n=4$; $p=0.049$ ). This is compatible with the partial displacement of TPMPA by a higher concentration of GABA in the synaptic cleft in $10 \mathrm{mM} \mathrm{Ca}^{2+}$. Thus, either spillover or multivesicular release does occur at inhibitory autapses in high $\mathrm{Ca}^{2+}$.

What are the implications of this finding for our $V-M$ analysis? By performing computer simulations, we show that cross talk, if it produces partial saturation of postsynaptic receptors, leads to an underestimate of the number, $N$, of independent release sites (supplemental material, available at www.jneurosci. org). However, the simulations also show that release probability, $P_{\mathrm{r}}$, is correctly estimated, despite errors in $N$ (next section).

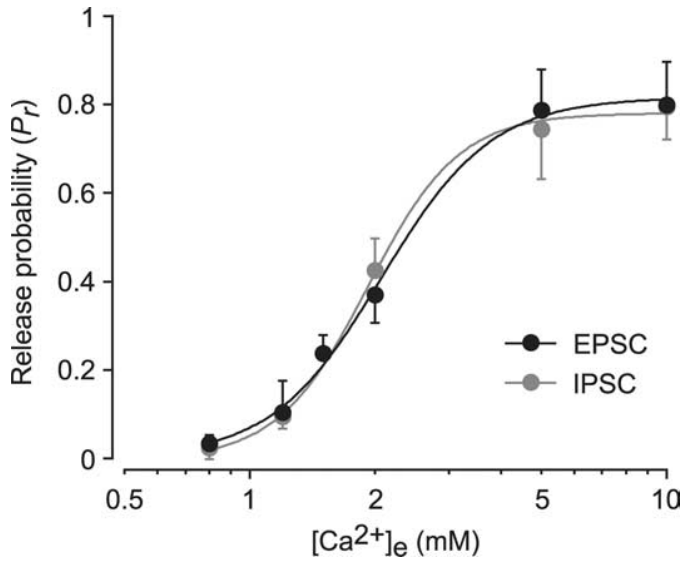

Figure 5. The plot of probability of transmitter release $\left(P_{r}\right)$ versus $\left[\mathrm{Ca}^{2+}\right]_{e}$ is indistinguishable for autaptic EPSCs and IPSCS. Each data point is the average across all cells ( $n=6$ for both EPSCS and IPSCS) of $P_{\mathrm{r}}$ calculated as described in Materials and Methods. $\left[\mathrm{Mg}^{2+}\right]_{\mathrm{e}}$ was always adjusted in the external solution so the total concentration of divalent cations $\left(\left[\mathrm{Ca}^{2+}\right]\right.$ plus $\left[\mathrm{Mg}^{2+}\right]$ ) was $11 \mathrm{~mm}$. Smooth curves are fits to the Hill equation (see Results). The curves for EPSCs and IPSCs are not significantly different.

\section{Dependence of $P_{\mathrm{r}}$ on $\left[\mathrm{Ca}^{2+}\right]_{\mathrm{e}}$ is the same at excitatory and inhibitory autapses}

Our $V-M$ analysis has provided estimates of $N$ and $Q$ for each cell. Hence, knowing $I$ in each $\left[\mathrm{Ca}^{2+}\right]_{\mathrm{e}}$, Equation 1 allows us to calculate $P_{\mathrm{r}}$ for each of these $\mathrm{Ca}^{2+}$ concentrations. Because $P_{\mathrm{r}}$ is calculated from a ratio of $I$ and $N$, both of which are equally reduced by any saturation resulting from cross talk, our estimate of $P_{\mathrm{r}}$ is little affected (supplemental material, available at www.jneurosci.org). Figure 5 shows the averaged plot of $P_{\mathrm{r}}$ versus $\left[\mathrm{Ca}^{2+}\right]_{\mathrm{e}}$ for excitatory and inhibitory autapses (black and gray symbols, respectively). The smooth curves are fits of the Hill equation (Eq. 5): excitatory (black curve), half-maximal concentration $\left(c_{1 / 2}\right)=$ $2.07 \pm 0.11 \mathrm{~mm}$, Hill coefficient $(h)=3.27 \pm 0.72$; inhibitory (gray curve), $c_{1 / 2}=1.92 \pm 0.09 \mathrm{~mm}, h=3.95 \pm 1.01$. These curves are not significantly different (Kolmogorov-Smirnov test, $p>0.1)$.

\section{$V-M$ analysis reveals different mechanisms of short-term depression at excitatory and inhibitory autapses}

All of our experiments used paired-pulse stimulation to elicit pairs of autaptic PSCs (Figs. 2A, 4A). So far we have focused exclusively on the first PSC of each pair. We next considered the properties of the second PSC.

The paired-pulse ratio (PPR; amplitude of the second PSC divided by that of the first) depended on $\left[\mathrm{Ca}^{2+}\right]_{\mathrm{e}}$ (Fig. $6 \mathrm{~B}$ ). This accords with the well known finding that PPR at least partially reflects $P_{\mathrm{r}}$ (Zucker and Regehr, 2002). However, whereas autaptic EPSCs showed facilitation at low $(<2 \mathrm{mM}) \mathrm{Ca}^{2+}$ concentrations, autaptic IPSCs always showed depression (Fig. 6A,B). This occurred despite the fact that the presynaptic stimulus, assayed as the PPR of peak $I_{\mathrm{Na}}$ during the stimulus step, was little affected by changes in $\left[\mathrm{Ca}^{2+}\right]_{\mathrm{e}}$ (Fig. $6 \mathrm{~B}$, gray symbols). This difference between EPSCs and IPSCs might be due to presynaptic factors; e.g., if $P_{\mathrm{r}}$ is higher for IPSCs in low $\left[\mathrm{Ca}^{2+}\right]_{\mathrm{e}}$, more vesicle depletion might occur on the first stimulus, leading to greater depression on the second (Zucker and Regehr, 2002). This hypothesis can be ruled out because we found that $P_{\mathrm{r}}$ is identical for EPSCs and IPSCs in the same $\left[\mathrm{Ca}^{2+}\right]_{\mathrm{e}}$ (Fig. 5). We also excluded the possibility that GABA spills out of the synaptic cleft at inhibitory contacts and activates presynaptic $\mathrm{GABA}_{\mathrm{B}}$ autoreceptors, inhibiting 
release: paired-pulse depression of IPSCs was unaffected by the $\mathrm{GABA}_{\mathrm{B}}$ receptor antagonist CGP 55845 (PPR for IPSCs measured in $10 \mu \mathrm{M}$ CGP divided by that under control conditions $=1.05 \pm 0.02, n=3$, not significantly different, $p>0.6$ ).

Another possible explanation is that paired-pulse depression might be due to a postsynaptic change in $Q$ (e.g., if greater desensitization occurs during the first IPSC, $Q$ will be smaller for the second; Zucker and Regehr, 2002). Finally, depression might result from a reduction in the number of release sites, $N$ (e.g., if a fraction of boutons is silenced following the first stimulus, perhaps because of propagation failures in the axon, $N$ will be smaller for the second stimulus). These hypotheses can be tested directly by $V-M$ analysis of the second PSC.

For EPSCs, the plot of $\sigma^{2}$ versus $I$ for the second EPSC was typically similar in shape to that of the first, except it did not curve over as far (Fig. 6C, left) (similar results in $n=5$ cells). This is the behavior expected for short-term plasticity with a mainly presynaptic locus of expression (Silver et al., 1998). In contrast, the corresponding plot for IPSCs showed a pronounced distortion for currents recorded in $\mathrm{Ca}^{2+}$ concentrations above $2 \mathrm{mM}$, with the points for the second IPSC being shifted strongly to the left of those for the first IPSC (Fig. 6C, right, shift indicated by gray arrow) (similar results in $n=4$ cells). What could give rise to this type of distortion? This question was addressed using a combination of simulations and experiments.
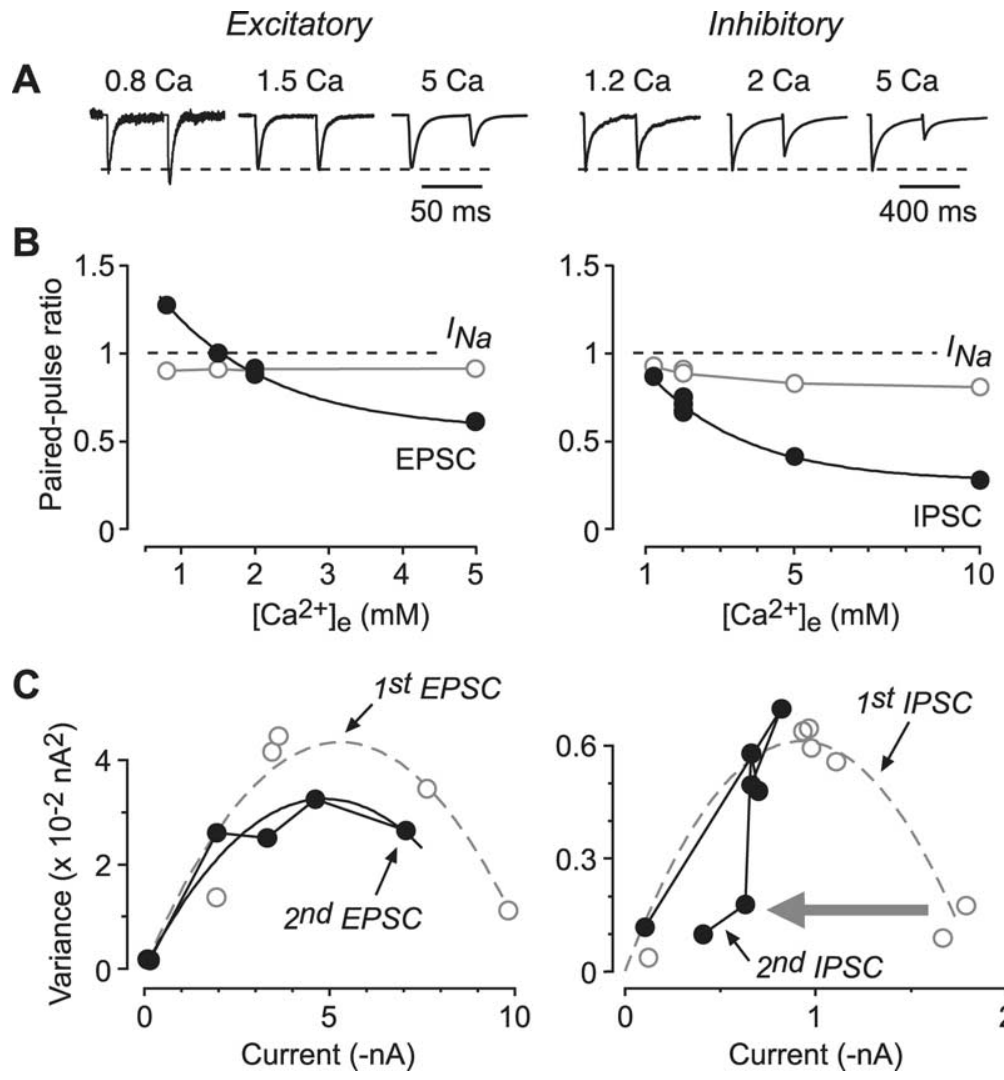

Figure 6. $\quad V-M$ analysis shows that short-term plasticity is largely due to presynaptic factors for autaptic EPSCs, but not for autaptic IPSCs. $A$, Typical examples of EPSC (left) and IPSC (right) paired-pulse responses in three different $\left[\mathrm{Ca}^{2+}\right]_{\mathrm{e}}$. Each trace has been normalized to the response to the first stimulus (dashed horizontal line). Stimulus transients have been blanked. $\boldsymbol{B}, \boldsymbol{B}$ Back symbols and lines plot the paired-pulse ratio (amplitude of the second autaptic response divided by the amplitude of the first) versus $\left[\mathrm{Ca}^{2+}\right]_{\mathrm{e}}$ for EPSCs (left) and IPSCs (right). Gray symbols and lines plot the paired-pulse ratio of peak $I_{\mathrm{Na}}$ evoked by the autaptic stimulus step in the same cells. EPSCs exhibit paired-pulse facilitation in low $\left[\mathrm{Ca}^{2+}\right]_{\mathrm{e}}$, whereas IPSCs always show paired-pulse depression. Each panel is from a single cell (same as in $\boldsymbol{A}$ ). $\boldsymbol{C}, \mathrm{V}-\mathrm{M}$ plots for the first and second EPSCs/IPSCs in the paired-pulse response (gray and black symbols, respectively) for EPSCs (left) and IPSCs (right). The smooth curves are parabola fits to the points. The parabola fit to the second EPSC indicates a presynaptic locus of short-term plasticity (i.e., change in $P_{r}$ ). The distortion observed for the IPSCs measured in 5 and $10 \mathrm{~mm}\left[\mathrm{Ca}^{2+}\right]_{\mathrm{e}}$ (large gray arrow) indicates that paired-pulse depression in these solutions is due to a change in either $N$ or $Q$ (see Fig. $7 A$ ). Data are from the same cells as in $\boldsymbol{A}$ and $\boldsymbol{B}$.

\section{Paired-pulse depression of IPSCs appears to involve a reduction in $N$}

Simulations were performed to explore the effect on the $V-M$ plot of changing either $Q$ or $N$ (Fig. $7 A$ ). If both $Q$ and $N$ are constant for all $P_{\mathrm{r}}$ values (Fig. $7 A$, dashed line, inset), the simulated $V-M$ plot is a parabola, as expected (Fig. $7 A$, dashed curve). If $Q$ decreases at higher $P_{\mathrm{r}}$ values (Fig. $7 A$, solid line, inset), perhaps due to postsynaptic desensitization in higher $\left[\mathrm{Ca}^{2+}\right]_{e}$, the $V-M$ plot shows a leftward distortion (Fig. 7A, black curve) similar to that observed experimentally for IPSCs (Fig. 6C, right). Alternatively, if $N$ decreases at higher $P_{\mathrm{r}}$ values, due to a loss of release sites in higher $\left[\mathrm{Ca}^{2+}\right]_{\mathrm{e}}$, the $V-M$ plot shows a similar distortion (Fig. 7A, gray curve). Thus, a $P_{\mathrm{r}}$-dependent reduction in either $Q$ or $N$ could account for the observed distortion of the $V-M$ plot for the second IPSC, but the effects are so similar that they are unlikely to be distinguishable from $V-M$ data alone (cf. Clements and Silver, 2000; Oleskevich et al., 2000). Therefore, we performed additional experiments to determine whether $Q$ or $N$ changes.

We used two approaches to test the hypothesis that $Q$ is reduced in paired-pulse depression of IPSCs. First, the low-affinity $\mathrm{GABA}_{\mathrm{A}}$ receptor antagonist, TPMPA, was used in a standard kind of experiment to look for desensitization of subsynaptic $\mathrm{GABA}_{\mathrm{A}}$ receptors (Kirischuk et al., 2002). If desensitization is important, application of TPMPA should, at least partially, reverse pairedpulse depression. However, this was not the case. IPSCs recorded in the presence of TPMPA $(200 \mu \mathrm{M})$ exhibited paired-pulse depression similar to IPSCs recorded both under control conditions and in the presence of a high-affinity $\mathrm{GABA}_{\mathrm{A}}$ receptor antagonist (gabazine, $80 \mathrm{nM}$ ) in $10 \mathrm{mM} \mathrm{Ca}^{2+}$ (control: $0.47 \pm 0.02$; TPMPA: $0.48 \pm 0.02$; gabazine: $0.47 \pm 0.02 ; p>0.5, n=7$; data not shown). These results suggest that $\mathrm{GABA}_{\mathrm{A}}$ receptor desensitization does not underlie paired-pulse depression at inhibitory autapses.

In a second experiment, we recorded asynchronous miniature IPSCs following paired-pulse stimulation in $10 \mathrm{mM} \mathrm{Ca}^{2+}$ (Fig. $7 B)$. If $Q$ is reduced, the mIPSC amplitudes should also be transiently reduced, recovering with the same time course as the recovery of IPSCs from paired-pulse depression (Fig. 7C,D). Again, this was not observed. Instead, the opposite effect was seen: mIPSC amplitudes were slightly elevated for up to $\sim 4 \mathrm{~s}$ following stimulation, compared with the mean amplitude of spontaneous mIPSCs recorded without preceding stimulation (Fig. 7C). This apparent increase in mIPSC amplitude was probably due to measurement errors: immediately after the stimulus in high calcium, asynchronous mIPSCs occur at a high rate, producing unresolv- 

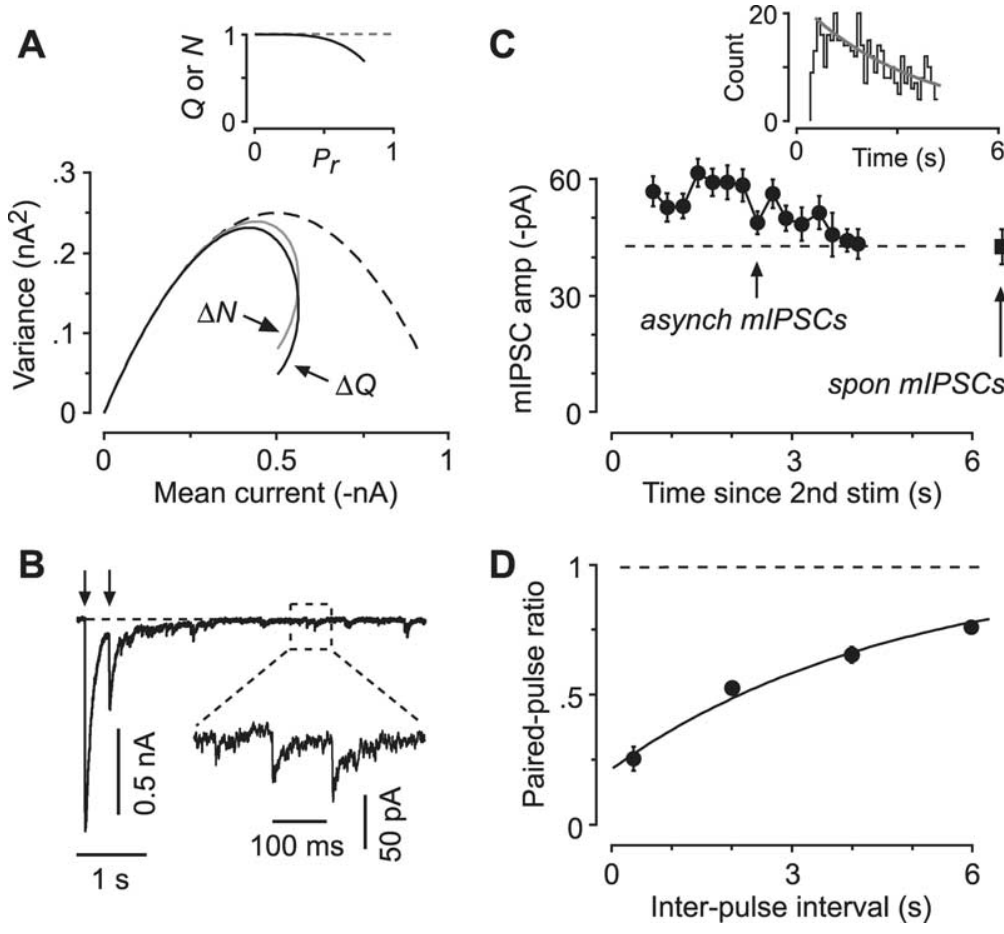

Figure 7. Simulations show that paired-pulse depression of IPSC in high $\left[\mathrm{Ca}^{2+}\right]_{\mathrm{e}}$ could be due to a change in either $N$ or $Q$, but measurements of asynchronous miniature IPSCS allow us to rule out a change in $\mathbf{Q}$. A, Simulated $V-M$ plots calculated for three different conditions: (1) $N=1, Q=1$ for all values of $P_{r}$ (dashed curve in main plot). (2) $N=1$ for all $P_{\mathrm{r}}$ values, but $Q$ varies with $P_{r}$ as shown in the inset (solid black curve in main plot, labeled " $\Delta Q^{\prime \prime}$ ). This could occur with desensitization of postsynaptic receptors for the second stimulation in higher $\left[\mathrm{Ca}^{2+}\right]_{\mathrm{e}}$. (3) $Q=1$ for all $P_{\mathrm{r}}$ values, but $N$ varies with $P_{\mathrm{r}}$ as shown in the inset (gray curve in main plot, labeled " $\left.\Delta N^{\prime \prime}\right)$. This could occur with loss of functional release sites for the second stimulation in higher $\left[\mathrm{Ca}^{2+}\right]_{\mathrm{e}}$. Changes in $\mathrm{Q}$ or $N$ produce similar distortion of the $V-M$ plot. This simulated distortion is similar to that seen experimentally (Fig. 6C, left). B, Paired-pulse autaptic IPSCs evoked by two stimuli (arrows) at a 350 ms interval in $10 \mathrm{~mm}\left[\mathrm{Ca}^{2+}\right]_{\mathrm{e}}$. Following the second IPSC is a flurry of asynchronous miniature IPSCs, shown expanded in the inset. Stimulus transients have been blanked. C, Mean amplitude of asynchronous mIPSCs versus time since the second autaptic stimulus (filled circles, $n=4$ cells). Means were calculated for all mIPSCs falling within a 250-ms-long time window around the indicated time delay. Also shown (filled square) is the mean amplitude of spontaneous mIPSCs captured in the same four cells in the absence of autaptic stimulation. Inset shows an averaged histogram of the frequency of occurrence of asynchronous mIPSCs versus time since the second autaptic stimulus, showing a decline in the frequency of mIPSCs over a similar time course as the decline in the mean amplitude of mIPSCS (main plot in this panel; same cells). This is consistent with the hypothesis that unresolved temporal overlap of mIPSCs occurring at higher frequencies is responsible for the apparently elevated mean mIPSC amplitude for a few seconds following the autaptic stimuli. $\boldsymbol{D}$, Mean paired-pulse ratio versus interpulse interval for evoked IPSCS, recorded in $10 \mathrm{~mm}\left[\mathrm{Ca}^{2+}\right]_{\mathrm{e}}$. Data were averaged from the same cells as in $\boldsymbol{C}$.

able temporal overlap and summation of individual events, raising the apparent mean amplitude of mIPSCs. Over the course of a few seconds the asynchronous mIPSCs decline in frequency (Fig. 7C, inset), resulting in fewer collisions and a more accurate estimation of individual mIPSC amplitudes. Moreover, it is difficult to think of a purely postsynaptic mechanism that can transiently increase $Q$ within a few hundred milliseconds of paired stimuli. In summary, these results confirm that a reduction in $Q$ is unlikely to contribute significantly to paired-pulse depression of IPSCs.

By a process of elimination, our results therefore suggest that paired-pulse depression of autaptic IPSCs is likely to involve a reduction in $N$.

\section{Discussion}

In this paper we have used the variance-mean technique to compare the quantal behavior of glutamatergic and GABAergic autapses in cultures prepared from region CA1 of rodent hippocampus. By taking advantage of the unique properties of this system, we validate the $V-M$ approach, showing that the simple version (Eq. 3) is adequate under our conditions because it is relatively insensitive to heterogeneities in release probability and quantal amplitude. We also use our results to calculate plots of mean $P_{\mathrm{r}}$ versus $\left[\mathrm{Ca}^{2+}\right]_{\mathrm{e}}$, showing that these plots are indistinguishable for excitatory and inhibitory autapses. Finally, we show that $V-M$ analysis allows us to identify factors involved in short-term synaptic plasticity, with clear differences between excitatory and inhibitory connections.

\section{Strengths and weaknesses of the $V-M$ approach}

$V-M$ analysis is increasingly more popular than classical histogram-based quantal analysis as a means of characterizing synaptic function (Silver et al., 1998; Reid and Clements, 1999; Oleskevich et al., 2000; Oleskevich and Walmsley, 2002; Foster and Regehr, 2004; Sargent et al., 2005; Biró et al., 2006; Zhang et al., 2006; Baldelli et al., 2007). A major advantage of the $V-M$ approach, at least in its simple form, is that estimates of $N$ and $Q$ can be obtained relatively easily, without the complex and error-prone histogram-fitting procedure of classical quantal analysis (Stricker and Redman, 2003; Ninio, 2007). The analytical simplicity of the $V-M$ method comes at the cost of more complex experiments: stable responses must be obtained from the same cell in a variety of external solutions that alter $P_{\mathrm{r}}$. Fewer responses need to be recorded in each of these solutions than is the case for classical quantal analysis [ 50-200 compared with many hundreds (Stricker and Redman, 2003)] because only the mean and variance are estimated for the $V-M$ approach, not a histogram of amplitudes. However, the total number of amplitude measurements in all solutions can still be large for the $V-M$ method, with challenging requirements for stability.

The formal assumptions underpinning both classical and $V-M$ methods of quantal analysis are similar (see Materials and Methods). How valid are these assumptions under the conditions of our experiments? It is assumed that the number $N$ of release sites stays constant as $P_{\mathrm{r}}$ is varied widely (Clements and Silver, 2000). This may not be the case if, for example, the propagation of presynaptic action potentials into axonal branches varies with changing $\left[\mathrm{Ca}^{2+}\right]_{\mathrm{e}}$. Indeed, we suggest that an effect of this kind may underlie paired-pulse depression of IPSCs in high $\left[\mathrm{Ca}^{2+}\right]_{\mathrm{e}}$ (Fig. 6). However, this is unlikely to be occurring for the first EPSC or IPSC of each pair, which is the focus of most of our analysis. We minimized the possibility of propagation failures in the axon by keeping the total concentration of divalent ions $\left(\left[\mathrm{Ca}^{2+}\right]_{\mathrm{e}}+\left[\mathrm{Mg}^{2+}\right]_{\mathrm{e}}\right)$ constant in our solutions, avoiding the effect of changing surface charge on action potential propagation (Frankenhaeuser and Hodgkin, 1957). In addition, we confirmed that the amplitude of the sodium action current, $I_{\mathrm{Na}}$, evoked by 
the autaptic stimulus (Figs. $2 B, 4 B$ ) was stable across all solutions.

The estimate of quantal amplitude, $Q$, obtained from $V-M$ analysis agreed closely with direct measurements of the miniature PSC amplitude, after correcting for latency jitter (Fig. 1). $\mathrm{N} \mathrm{ob-}$ tained from our analysis could not be compared with the number of immunolabeled boutons, due to technical limitations. In any case, such a comparison may not be appropriate. $N$ is commonly equated to the number of morphologically identified synaptic release sites (Redman, 1990), but the presence of cross talk between release sites (Tong and Jahr, 1994; this study) suggests that the morphological number of terminals may underestimate the number $N$ of independent release sites. However, this reinterpretation of $N$ does not invalidate the estimates of $P_{\mathrm{r}}$ (supplemental material, available at www.jneurosci.org).

In common with many culture experiments, our experiments were conducted at room temperature, but recent work has highlighted the complex changes in synaptic transmission that occur when the temperature is raised to near-physiological levels. For instance, it has been suggested that $P_{\mathrm{r}}$ at glutamatergic autapses is reduced by $\sim 36 \%$ when the temperature is increased from $25^{\circ} \mathrm{C}$ to $35^{\circ} \mathrm{C}$ (Pyott and Rosenmund, 2002). Changes in paired-pulse facilitation (Klyachko and Stevens, 2006) and in vesicle recycling kinetics during high-frequency trains (Fernández-Alfonso and Ryan, 2004; Micheva and Smith, 2005; Balaji et al., 2008) have also been reported with elevations in temperature. Because our work focuses on a comparison between excitatory and inhibitory neurotransmission, and does not use high-frequency trains, concerns about temperature effects on our conclusions are blunted. Nevertheless, future work should ideally be conducted under more physiological conditions.

\section{Properties of excitatory and inhibitory autapses}

We have compared the quantal properties of excitatory and inhibitory neurotransmission under identical conditions in hippocampal cultures. Most quantal analysis has focused on the properties of the much more numerous excitatory synapses (Bekkers and Stevens, 1995; Biró et al., 2005; Tyler et al., 2006) (cf. Baldelli et al., 2007), but there is evidence that the release properties of inhibitory synapses are different (Terada et al., 1999; Moulder et al., 2007; Sakaba, 2008). Moreover, GABAergic interneurons in the hippocampus exhibit considerable functional diversity (McBain and Fisahn, 2001). Some of this diversity is preserved in the different firing properties (single- vs multi-spiking) (Fig. 3C) and differential expression of molecular markers (our unpublished data) in our cultures. However, the diversity is less than observed in vivo, suggesting that subtleties may be lost in vitro. Hence, we can only make a broad comparison between generic inhibitory and excitatory neurons in culture.

Both excitatory and inhibitory neurotransmission are well described by the simple version of $V-M$ analysis when the first PSCs in a train are analyzed (Figs. 2, 4). Both predict a mean $Q$ that agrees well with the mean amplitude of miniature synaptic currents measured in the same cell, as expected, although for inhibitory neurons $Q$ is slightly smaller. This may be due to the greater noise we encountered in inhibitory cell recordings, which is likely to obscure smaller mIPSCs. These results suggest that the assumptions underlying $V-M$ analysis are equally applicable to both types of synapse, at least when PSCs are evoked at low frequency.

We found that the dependence of $P_{\mathrm{r}}$ on $\left[\mathrm{Ca}^{2+}\right]_{\mathrm{e}}$ is nearly identical for both excitatory and inhibitory autapses (Fig. 5), despite some reported differences in presynaptic vesicular proteins
(Terada et al., 1999; Gitler et al., 2004). The estimated values of half-maximal $\left[\mathrm{Ca}^{2+}\right]_{\mathrm{e}}\left(c_{1 / 2} \sim 2 \mathrm{mM}\right)$ and Hill coefficient $(h=$ 3-4) are similar to values obtained from plots of PSC amplitude versus $\left[\mathrm{Ca}^{2+}\right]_{\mathrm{e}}$ for synapses in hippocampal slices (Baldelli et al., 2005). Our approach differs in that it provides an estimate of absolute $P_{\mathrm{r}}$ rather than normalized current amplitude. We find that $P_{\mathrm{r}}$ asymptotes at $\sim 0.8$, rather than unity, indicating that a saturating concentration of $\left[\mathrm{Ca}^{2+}\right]_{\mathrm{e}}\left(10 \mathrm{mM}\right.$ plus $\left.1 \mathrm{mM} \mathrm{Mg}^{2+}\right)$ enables $\sim 80 \%$ of release sites to release a quantum of neurotransmitter.

Although we found similar quantal properties for excitatory and inhibitory autapses when postsynaptic responses are evoked at low frequency $(0.1-0.2 \mathrm{~Hz}), V-M$ analysis of paired-pulse responses (interpulse interval $50 \mathrm{~ms}$ for EPSCs, $350 \mathrm{~ms}$ for IPSCs) revealed differing mechanisms of short-term synaptic plasticity in the two cell types. For the second of two excitatory PSCs, the $V-M$ plot at high $\left[\mathrm{Ca}^{2+}\right]_{\mathrm{e}}$ was altered in a way that indicated a reduction in $P_{\mathrm{r}}$ with little effect on $Q$ or $N$. In contrast, the $V-M$ plot for the second of two inhibitory PSCs was distorted in a manner indicative of changes in either $Q$ (Clements and Silver, 2000) or N. Two lines of evidence allowed us to exclude a change in $Q$, leading to our conclusion that a change in $N$ is responsible. The locus of short-term depression of IPSCs is controversial, with evidence for both presynaptic (Davies et al., 1990; Jensen et al., 1999) and postsynaptic (Kirischuk et al., 2002; Brager et al., 2003) mechanisms. Our novel suggestion of changes in $N$ points to a temporary, $P_{\mathrm{r}}$-dependent loss of functional release sites, perhaps due to branch point failures of action potential propagation in the axons of inhibitory neurons (Grossman et al., 1979). This hypothesis would need to be explicitly tested in future experiments.

\section{Conclusions}

By taking advantage of a convenient culture system for the study of synaptic transmission, we have presented a detailed comparison of glutamatergic and GABAergic neurotransmission under identical recording conditions using the variance-mean method of quantal analysis. We found a number of similarities between these two types of synapse, but have also found that they differ in the mechanisms responsible for paired-pulse depression under high- $P_{\mathrm{r}}$ conditions. Although the high- $P_{\mathrm{r}}$ condition applied here $\left(10 \mathrm{mM} \mathrm{Ca}^{2+}\right)$ is unlikely to be encountered physiologically, our findings do draw attention to latent biophysical differences between excitation and inhibition that may become manifest under some circumstances. We conclude that the basic functional properties of excitatory and inhibitory neurotransmission in our system are remarkably similar at near-physiological levels of $\left[\mathrm{Ca}^{2+}\right]_{\mathrm{e}}$. This suggests that, despite differences in detail, the two types of synapse have evolved to provide matched functionality for normal brain operation.

\section{References}

Balaji J, Armbruster M, Ryan TA (2008) Calcium control of endocytic capacity at a CNS synapse. J Neurosci 28:6742-6749.

Baldelli P, Hernandez-Guijo JM, Carabelli V, Carbone E (2005) Brainderived neurotrophic factor enhances GABA release probability and nonuniform distribution of $\mathrm{N}$ - and $\mathrm{P} / \mathrm{Q}$-type channels on release sites of hippocampal inhibitory synapses. J Neurosci 25:3358-3368.

Baldelli P, Fassio A, Valtorta F, Benfenati F (2007) Lack of synapsin I reduces the readily releasable pool of synaptic vesicles at central inhibitory synapses. J Neurosci 27:13520-13531.

Bekkers JM (2005) Presynaptically silent GABA synapses in hippocampus. J Neurosci 25:4031-4039.

Bekkers JM, Stevens CF (1995) Quantal analysis of EPSCs recorded from 
small numbers of synapses in hippocampal cultures. J Neurophysiol 73:1145-1156.

Bekkers JM, Richerson GB, Stevens CF (1990) Origin of variability in quantal size in cultured hippocampal neurons and hippocampal slices. Proc Natl Acad Sci U S A 87:5359-5362.

Biró AA, Holderith NB, Nusser Z (2005) Quantal size is independent of the release probability at hippocampal excitatory synapses. J Neurosci 25:223-232.

Biró AA, Holderith NB, Nusser Z (2006) Release probability-dependent scaling of the postsynaptic responses at single hippocampal GABAergic synapses. J Neurosci 26:12487-12496.

Brager DH, Luther PW, Erdélyi F, Szabó G, Alger BE (2003) Regulation of exocytosis from single visualized GABAergic boutons in hippocampal slices. J Neurosci 23:10475-10486.

Christie JM, Jahr CE (2006) Multivesicular release at Schaffer collateralCA1 hippocampal synapses. J Neurosci 26:210-216.

Clements JD, Silver RA (2000) Unveiling synaptic plasticity: a new graphical and analytical approach. Trends Neurosci 23:105-113.

Davies CH, Davies SN, Collingridge GL (1990) Paired-pulse depression of monosynaptic GABA-mediated inhibitory postsynaptic responses in rat hippocampus. J Physiol 424:513-531.

Del Castillo J, Katz B (1954) Quantal components of the end-plate potential. J Physiol 124:560-573.

Diamond JS, Jahr CE (1995) Asynchronous release of synaptic vesicles determines the time course of the AMPA receptor-mediated EPSC. Neuron 15:1097-1107.

Fedulova SA, Vasilyev DV, Isaeva EV, Romanyuk SG, Veselovsky NS (1999) Possibility of multiquantal transmission at single inhibitory synapse in cultured rat hippocampal neurons. Neuroscience 92:1217-1230.

Fernández-Alfonso T, Ryan TA (2004) The kinetics of synaptic vesicle pool depletion at CNS synaptic terminals. Neuron 41:943-953.

Forti L, Bossi M, Bergamaschi A, Villa A, Malgaroli A (1997) Loose-patch recordings of single quanta at individual hippocampal synapses. Nature 388:874-878.

Foster KA, Regehr WG (2004) Variance-mean analysis in the presence of a rapid antagonist indicates vesicle depletion underlies depression at the climbing fiber synapse. Neuron 43:119-131.

Frankenhaeuser B, Hodgkin AL (1957) The action of calcium on the electrical properties of squid axons. J Physiol 137:218-244.

Gitler D, Takagishi Y, Feng J, Ren Y, Rodriguiz RM, Wetsel WC, Greengard P, Augustine GJ (2004) Different presynaptic roles of synapsins at excitatory and inhibitory synapses. J Neurosci 24:11368-11380.

Grossman Y, Parnas I, Spira ME (1979) Ionic mechanisms involved in differential conduction of potentials at high frequency in a branching axon. J Physiol 295:307-322.

Hartman KN, Pal SK, Burrone J, Murthy VN (2006) Activity-dependent regulation of inhibitory synaptic transmission in hippocampal neurons. Nat Neurosci 9:642-649.

Jensen K, Lambert JD, Jensen MS (1999) Activity-dependent depression of GABAergic IPSCs in cultured hippocampal neurons. J Neurophysiol $82: 42-49$.

Kirischuk S, Clements JD, Grantyn R (2002) Presynaptic and postsynaptic mechanisms underlie paired pulse depression at single GABAergic boutons in rat collicular cultures. J Physiol 543:99-116.

Klyachko VA, Stevens CF (2006) Temperature-dependent shift of balance among the components of short-term plasticity in hippocampal synapses. J Neurosci 26:6945-6957.

Liu G, Choi S, Tsien RW (1999) Variability of neurotransmitter concentration and nonsaturation of postsynaptic AMPA receptors at synapses in hippocampal cultures and slices. Neuron 22:395-409.

McBain CJ, Fisahn A (2001) Interneurons unbound. Nat Rev Neurosci 2:11-23.

Micheva KD, Smith SJ (2005) Strong effects of subphysiological temperature on the function and plasticity of mammalian presynaptic terminals. J Neurosci 25:7481-7488.

Moulder KL, Jiang X, Taylor AA, Shin W, Gillis KD, Mennerick S (2007) Vesicle pool heterogeneity at hippocampal glutamate and GABA synapses. J Neurosci 27:9846-9854.
Murthy VN, Sejnowski TJ, Stevens CF (1997) Heterogeneous release properties of visualized individual hippocampal synapses. Neuron 18:599-612.

Neher E, Sakaba T (2001) Combining deconvolution and noise analysis for the estimation of transmitter release rates at the Calyx of Held. J Neurosci 21:444-461.

Ninio J (2007) Doubts about quantal analysis. J Neurophysiol 98:1827-1835; author reply 1836-1840.

Oleskevich S, Walmsley B (2002) Synaptic transmission in the auditory brainstem of normal and congenitally deaf mice. J Physiol 540:447-455.

Oleskevich S, Clements J, Walmsley B (2000) Release probability modulates short-term plasticity at a rat giant terminal. J Physiol 524:513-523.

Pyott SJ, Rosenmund C (2002) The effects of temperature on vesicular supply and release in autaptic cultures of rat and mouse hippocampal neurons. J Physiol 539:523-535.

Redman SJ (1990) Quantal analysis of synaptic potentials in neurons of the central nervous system. Physiol Rev 70:165-198.

Reid CA, Clements JD (1999) Postsynaptic expression of long-term potentiation in the rat dentate gyrus demonstrated by variance-mean analysis. J Physiol 518:121-130.

Rosenmund C, Clements JD, Westbrook GL (1993) Nonuniform probability of glutamate release at a hippocampal synapse. Science 262:754-757.

Sakaba T (2008) Two $\mathrm{Ca}^{2+}$-dependent steps controlling synaptic vesicle fusion and replenishment at the cerebellar basket cell terminal. Neuron 57:406-419.

Sargent PB, Saviane C, Nielsen TA, DiGregorio DA, Silver RA (2005) Rapid vesicular release, quantal variability, and spillover contribute to the precision and reliability of transmission at a glomerular synapse. J Neurosci 25:8173-8187.

Saviane C, Silver RA (2006) Errors in the estimation of the variance: implications for multiple-probability fluctuation analysis. J Neurosci Methods 153:250-260.

Silver RA (2003) Estimation of nonuniform quantal parameters with multiple-probability fluctuation analysis: theory, application and limitations. J Neurosci Methods 130:127-141.

Silver RA, Momiyama A, Cull-Candy SG (1998) Locus of frequencydependent depression identified with multiple-probability fluctuation analysis at rat climbing fibre-Purkinje cell synapses. J Physiol 510:881-902.

Singer JH, Berger AJ (1999) Contribution of single-channel properties to the time course and amplitude variance of quantal glycine currents recorded in rat motoneurons. J Neurophysiol 81:1608-1616.

Spruston N, McBain C (2007) Structural and functional properties of hippocampal neurons. In: The hippocampus book (Andersen P, Morris R, Amaral D, Bliss T, O’Keefe J, eds), pp 133-201. New York: Oxford UP.

Stricker C, Redman SJ (2003) Quantal analysis based on density estimation. J Neurosci Methods 130:159-171.

Tamamaki N, Yanagawa Y, Tomioka R, Miyazaki J, Obata K, Kaneko T (2003) Green fluorescent protein expression and colocalization with calretinin, parvalbumin, and somatostatin in the GAD67-GFP knock-in mouse. J Comp Neurol 467:60-79.

Terada S, Tsujimoto T, Takei Y, Takahashi T, Hirokawa N (1999) Impairment of inhibitory synaptic transmission in mice lacking synapsin I. J Cell Biol 145:1039-1048.

Tong G, Jahr CE (1994) Multivesicular release from excitatory synapses of cultured hippocampal neurons. Neuron 12:51-59.

Tyler WJ, Zhang XL, Hartman K, Winterer J, Muller W, Stanton PK, PozzoMiller L (2006) BDNF increases release probability and the size of a rapidly recycling vesicle pool within rat hippocampal excitatory synapses. J Physiol 574:787-803.

Zhang XL, Zhou ZY, Winterer J, Müller W, Stanton PK (2006) NMDAdependent, but not group I metabotropic glutamate receptor-dependent, long-term depression at Schaffer collateral-CA1 synapses is associated with long-term reduction of release from the rapidly recycling presynaptic vesicle pool. J Neurosci 26:10270-10280.

Zucker RS, Regehr WG (2002) Short-term synaptic plasticity. Annu Rev Physiol 64:355-405. 